IJMMS 27:11 (2001) 653-662

PII. S0161171201007244

http://ijmms.hindawi.com

(c) Hindawi Publishing Corp.

\title{
CONVERGENCE THEOREMS OF THE SEQUENCE OF ITERATES FOR A FINITE FAMILY ASYMPTOTICALLY NONEXPANSIVE MAPPINGS
}

\author{
JUI-CHI HUANG
}

(Received 30 March 2001)

\begin{abstract}
Let $E$ be a uniformly convex Banach space, $C$ a nonempty closed convex subset of $E$. In this paper, we introduce an iteration scheme with errors in the sense of Xu (1998) generated by $\left\{T_{j}: C \rightarrow C\right\}_{j=1}^{r}$ as follows: $U_{n(j)}=a_{n(j)} I+b_{n(j)} T_{j}^{n} U_{n(j-1)}+c_{n(j)} u_{n(j)}, j=$ $1,2, \ldots, r, x_{1} \in C, x_{n+1}=a_{n(r)} x_{n}+b_{n(r)} T_{r}^{n} U_{n(r-1)} x_{n}+c_{n(r)} u_{n(r)}, n \geq 1$, where $U_{n(0)}:=$ $I, I$ the identity map; and $\left\{u_{n(j)}\right\}$ are bounded sequences in $C$; and $\left\{a_{n(j)}\right\},\left\{b_{n(j)}\right\}$, and $\left\{c_{n(j)}\right\}$ are suitable sequences in $[0,1]$. We first consider the behaviour of iteration scheme above for a finite family of asymptotically nonexpansive mappings. Then we generalize theorems of Schu and Rhoades.
\end{abstract}

2000 Mathematics Subject Classification. 47H10.

1. Introduction. Let $C$ be a nonempty convex subset of a Banach space $E$. A mapping $T: C \rightarrow C$ is called asymptotically nonexpansive with sequence $\left\{k_{n}\right\}_{n=1}^{\infty}$ if $k_{n} \geq 1$ and $\lim _{n \rightarrow \infty} k_{n}=1$ such that

$$
\left\|T^{n} x-T^{n} y\right\| \leq k_{n}\|x-y\|
$$

for all $x, y \in C$ and all $n \in \mathbb{N}$. $T$ is called uniformly L-Lipschitzian if

$$
\left\|T^{n} x-T^{n} y\right\| \leq L\|x-y\|
$$

for all $x, y \in C$ and all $n \in \mathbb{N}$. It is clear that every asymptotically nonexpansive mapping is also uniformly $L$-Lipschitzian for some $L>0$. In [7], Schu introduced the modified Ishikawa iteration method as

$$
x_{n+1}=\alpha_{n} T^{n}\left(\beta_{n} T^{n} x_{n}+\left(1-\beta_{n}\right) x_{n}\right)+\left(1-\alpha_{n}\right) x_{n}, \quad n=1,2, \ldots,
$$

where $\left\{\alpha_{n}\right\}$ and $\left\{\beta_{n}\right\}$ are suitable sequences in $[0,1]$ and the modified Mann iteration method as

$$
x_{n+1}=\alpha_{n} T^{n} x_{n}+\left(1-\alpha_{n}\right) x_{n}, \quad n=1,2, \ldots,
$$

where $\left\{\alpha_{n}\right\}$ is a suitable sequence in $[0,1]$.

Using the iteration method (1.4), Schu [9, Lemma 1.5] and Rhoades [6, Theorem 1] obtained the following result: let $C$ be a bounded closed convex subset of a uniformly 
convex Banach space $E, T: C \rightarrow C$ an asymptotically nonexpansive mapping with sequence $\left\{k_{n}\right\}$ such that $\sum_{n=1}^{\infty}\left(k_{n}-1\right)<\infty$, and $\left\{\alpha_{n}\right\}$ a sequence in $[0,1]$ satisfying the condition $\varepsilon \leq \alpha_{n} \leq 1-\varepsilon$ for all $n \in \mathbb{N}$ and some $\varepsilon>0$. Suppose that $x_{1} \in C$ and that $\left\{x_{n}\right\}$ is given by (1.4). Then $\lim _{n \rightarrow \infty}\left\|x_{n}-T x_{n}\right\|=0$.

Note that $\sum_{n=1}^{\infty}\left(k_{n}-1\right)<\infty$ if and only if $\sum_{n=1}^{\infty}\left(k_{n}^{s}-1\right)<\infty$ for some $s>1$ (see [5, Remark 3]).

Let $C$ be a nonempty convex subset of a Banach space $E$. Let $T_{j}: C \rightarrow C$ be a given mapping for each $j=1,2, \ldots, r$. We now introduce an iteration scheme with errors in the sense of $\mathrm{Xu}[11]$ generated by $T_{1}, T_{2}, \ldots, T_{r}$ as follows: let $U_{n(0)}=I$, where $I$ is the identity map,

$$
\begin{aligned}
U_{n(1)} & =a_{n(1)} I+b_{n(1)} T_{1}^{n} U_{n(0)}+c_{n(1)} u_{n(1)}, \\
U_{n(2)} & =a_{n(2)} I+b_{n(2)} T_{2}^{n} U_{n(1)}+c_{n(2)} u_{n(2)}, \\
\vdots & \\
U_{n(r)} & =a_{n(r)} I+b_{n(r)} T_{r}^{n} U_{n(r-1)}+c_{n(r)} u_{n(r)}, \\
x_{n+1} & =a_{n(r)} x_{n}+b_{n(r)} T_{r}^{n} U_{n(r-1)} x_{n}+c_{n(r)} u_{n(r)}, \quad n \geq 1 .
\end{aligned}
$$

Here, $\left\{u_{n(j)}\right\}_{n=1}^{\infty}$ is a bounded sequence in $C$ for each $j=1,2, \ldots, r$, and $\left\{a_{n(j)}\right\}_{n=1}^{\infty}$, $\left\{b_{n(j)}\right\}_{n=1}^{\infty}$, and $\left\{c_{n(j)}\right\}_{n=1}^{\infty}$ are sequences in $[0,1]$ satisfying the conditions

$$
a_{n(j)}+b_{n(j)}+c_{n(j)}=1
$$

for all $n \in \mathbb{N}$ and each $j=1,2, \ldots, r$. This scheme contains the modified Mann and Ishikawa iteration methods with errors in the sense of Xu [11] (cf. [5]): for $r=1$, our scheme reduces to Mann-Xu type iteration and for $r=2, T_{1}=T_{2}$ to Ishikawa-Xu type iteration.

In 1972, Goebel and Kirk [1] proved that if $C$ is a bounded closed convex subset of a uniformly convex Banach space $E$, then every asymptotically nonexpansive selfmapping $T$ of $C$ has a fixed point. After the existence theorem of Goebel and Kirk [1], several authors including Schu [7, 9], Rhoades [6], Huang [3] and Osilike and Aniagbosor [5] have studied methods for the iterative approximation of fixed points of asymptotically nonexpansive mappings. In this paper, we first extend the result above of [9, Lemma 1.5] and [6, Theorem 1] to the iteration scheme (1.5) and without the restrictions that $C$ is bounded. Then, using this result, we generalize [9, Theorems 2.1, 2.2, and 2.4] and [6, Theorems 2 and 3].

In the sequel, we will need the following results.

LEMMA 1.1 (see [5, Lemma 1]). Let $\left\{a_{n}\right\}_{n=1}^{\infty},\left\{b_{n}\right\}_{n=1}^{\infty}$, and $\left\{\delta_{n}\right\}_{n=1}^{\infty}$ be sequences of nonnegative real numbers satisfying the inequality

$$
a_{n+1} \leq\left(1+\delta_{n}\right) a_{n}+b_{n}, \quad n \geq 1 .
$$

If $\sum_{n=1}^{\infty} \delta_{n}<\infty$ and $\sum_{n=1}^{\infty} b_{n}<\infty$, then $\lim _{n \rightarrow \infty} a_{n}$ exists. In particular, if $\left\{a_{n}\right\}_{n=1}^{\infty}$ has a subsequence which converges strongly to zero, then $\lim _{n \rightarrow \infty} a_{n}=0$. 
LEMмA 1.2 (see [8, Lemma 2]). Let $\left\{\beta_{n}\right\}_{n=1}^{\infty}$ and $\left\{\omega_{n}\right\}_{n=1}^{\infty}$ be sequences of nonnegative numbers such that for some real numbers $N_{0} \geq 1$,

$$
\beta_{n+1} \leq\left(1-\delta_{n}\right) \beta_{n}+\omega_{n}
$$

for all $n \geq N_{0}$, where $\delta_{n} \in[0,1]$. If $\sum_{n=1}^{\infty} \delta_{n}=\infty$ and $\sum_{n=1}^{\infty} \omega_{n}<\infty$, then $\lim _{n \rightarrow \infty} \beta_{n}=0$.

THEOREM 1.3 (see [10, Theorem 2]). Let E be a uniformly convex Banach space and $r>0$. Then there exists a continuous, strictly increasing and convex function $g: \mathbb{R}^{+} \rightarrow$ $\mathbb{R}^{+}$such that $g(0)=0$ and

$$
\|\lambda x+(1-\lambda) y\|^{2} \leq \lambda\|x\|^{2}+(1-\lambda)\|y\|^{2}-\lambda(1-\lambda) g(\|x-y\|)
$$

for all $x, y \in B_{r}:=\{x \in E:\|x\| \leq r\}$ and $\lambda \in[0,1]$.

A Banach space $E$ is said to satisfy Opial's condition [4] if $x_{n} \rightarrow x$ weakly and $x \neq y$ imply

$$
\liminf _{n \rightarrow \infty}\left\|x_{n}-x\right\|<\liminf _{n \rightarrow \infty}\left\|x_{n}-y\right\|
$$

LEMMA 1.4 (see [2, Lemma 4]). Let E be a uniformly convex Banach space satisfying Opial's condition and $C$ a nonempty closed convex subset of E. Let $T: C \rightarrow C$ be an asymptotically nonexpansive mapping. Then $(I-T)$ is demiclosed at zero, that is, for each sequence $\left\{x_{n}\right\}$ in $C$, the conditions $x_{n} \rightarrow x$ weakly and $(I-T) x_{n} \rightarrow 0$ strongly imply $(I-T) x=0$.

2. Main results. For abbreviation, we denote the set of fixed points of a mapping $T$ by $F(T)$, and now prove the following results.

THEOREM 2.1. Let $C$ be a nonempty closed convex subset of a uniformly convex Banach space $E$ and $T_{j}: C \rightarrow C$ an asymptotically nonexpansive mapping with sequence $\left\{k_{n(j)}\right\}_{n=1}^{\infty}$ for each $j=1,2, \ldots, r$ such that $\sum_{n=1}^{\infty}\left(k_{n}-1\right)<\infty$, where $k_{n}:=$ $\max _{1 \leq j \leq r}\left\{k_{n(j)}\right\} \geq 1$ and $\cap_{j=1}^{r} F\left(T_{j}\right) \neq \varnothing$. Let $\left\{u_{n(j)}\right\}_{n=1}^{\infty}$ be a bounded sequence in $C$ for each $j=1,2, \ldots, r$ and let $\left\{a_{n(j)}\right\}_{n=1}^{\infty},\left\{b_{n(j)}\right\}_{n=1}^{\infty}$, and $\left\{c_{n(j)}\right\}_{n=1}^{\infty}$ be sequences in $[0,1]$ satisfying the conditions:

(i) $a_{n(j)}+b_{n(j)}+c_{n(j)}=1$ for all $n \in \mathbb{N}$ and each $j=1,2, \ldots, r$;

(ii) $\sum_{n=1}^{\infty} c_{n(j)}<\infty$ for each $j=1,2, \ldots, r$;

(iii) $0<a \leq \alpha_{n(j)} \leq b<1$ for all $n \in \mathbb{N}$, each $j=1,2, \ldots, r$, and some constants $a$, $b$, where $\alpha_{n(j)}:=b_{n(j)}+c_{n(j)}$.

Suppose that $\left\{x_{n}\right\}$ is given by (1.5). Then $\lim _{n \rightarrow \infty}\left\|x_{n}-T_{j} x_{n}\right\|=0$ for each $j=1,2, \ldots, r$.

In order to prove Theorem 2.1, we first prove the following lemmas.

LEMMA 2.2. Let $C$ be a nonempty convex subset of a Banach space $E$. Let $T_{j}: C \rightarrow C$ be a uniformly L-Lipschitzian mapping for each $j=1,2, \ldots, r$, and let $\left\{x_{n}\right\}$ be as in (1.5). 
Set $e_{n(j)}:=\left\|x_{n}-T_{j}^{n} U_{n(j-1)} x_{n}\right\|$ for all $n, j \in \mathbb{N}$. Then for all $n \geq 2$,

$$
\begin{aligned}
\left\|x_{n}-T_{1} x_{n}\right\| \leq & e_{n(1)}+\left(L^{2}+L\right) e_{n-1(r)}+L e_{n-1(1)}+\left(L^{2}+L\right) c_{n-1(r)}\left\|u_{n-1(r)}-x_{n-1}\right\|, \\
\left\|x_{n}-T_{j} x_{n}\right\| \leq & e_{n(j)}+\left(L^{2}+L\right) e_{n-1(r)}+L^{2} e_{n(j-1)}+L^{2} e_{n-1(j-1)}+L e_{n-1(j)} \\
& +\left(L^{2}+L\right) c_{n-1(r)}\left\|u_{n-1(r)}-x_{n-1}\right\|+L^{2} c_{n(j-1)}\left\|u_{n(j-1)}-x_{n}\right\| \\
& +L^{2} c_{n-1(j-1)}\left\|x_{n-1}-u_{n-1(j-1)}\right\|
\end{aligned}
$$

for each $j=2,3, \ldots, r$.

Proof. Observe that for $j=2,3, \ldots, r$ we have

$$
\begin{aligned}
\left\|U_{n(j-1)} x_{n}-U_{n-1(j-1)} x_{n-1}\right\| & \\
= & \|\left(a_{n(j-1)} x_{n}+b_{n(j-1)} T_{j-1}^{n} U_{n(j-2)} x_{n}+c_{n(j-1)} u_{n(j-1)}\right) \\
& -\left(a_{n-1(j-1)} x_{n-1}+b_{n-1(j-1)} T_{j-1}^{n-1} U_{n-1(j-2)} x_{n-1}\right. \\
& \left.+c_{n-1(j-1)} u_{n-1(j-1)}\right) \| \\
= & \|\left(x_{n}-x_{n-1}\right)+b_{n(j-1)}\left(T_{j-1}^{n} U_{n(j-2)} x_{n}-x_{n}\right) \\
& +c_{n(j-1)}\left(u_{n(j-1)}-x_{n}\right)+b_{n-1(j-1)}\left(x_{n-1}-T_{j-1}^{n-1} U_{n-1(j-2)} x_{n-1}\right) \\
& +c_{n-1(j-1)}\left(x_{n-1}-u_{n-1(j-1)}\right) \| \\
\leq & \left\|x_{n}-x_{n-1}\right\|+e_{n(j-1)}+e_{n-1(j-1)}+c_{n(j-1)}\left\|u_{n(j-1)}-x_{n}\right\| \\
& +c_{n-1(j-1)}\left\|x_{n-1}-u_{n-1(j-1)}\right\|, \\
\left\|x_{n}-x_{n-1}\right\|= & \left\|a_{n-1(r)} x_{n-1}+b_{n-1(r)} T_{r}^{n-1} U_{n-1(r-1)} x_{n-1}+c_{n-1(r)} u_{n-1(r)}-x_{n-1}\right\| \\
\leq & b_{n-1(r)}\left\|T_{r}^{n-1} U_{n-1(r-1)} x_{n-1}-x_{n-1}\right\|+c_{n-1(r)}\left\|u_{n-1(r)}-x_{n-1}\right\| \\
\leq & e_{n-1(r)}+c_{n-1(r)}\left\|u_{n-1(r)}-x_{n-1}\right\| .
\end{aligned}
$$

Therefore,

$$
\begin{aligned}
\left\|x_{n}-T_{j} x_{n}\right\| \leq & \left\|x_{n}-T_{j}^{n} U_{n(j-1)} x_{n}\right\|+\left\|T_{j}^{n} U_{n(j-1)} x_{n}-T_{j} x_{n}\right\| \\
\leq & e_{n(j)}+L\left\|T_{j}^{n-1} U_{n(j-1)} x_{n}-x_{n}\right\| \\
\leq & e_{n(j)}+L\left\|T_{j}^{n-1} U_{n(j-1)} x_{n}-T_{j}^{n-1} U_{n-1(j-1)} x_{n-1}\right\| \\
& +L\left\|T_{j}^{n-1} U_{n-1(j-1)} x_{n-1}-x_{n-1}\right\|+L\left\|x_{n-1}-x_{n}\right\| \\
\leq & e_{n(j)}+L^{2}\left\|U_{n(j-1)} x_{n}-U_{n-1(j-1)} x_{n-1}\right\| \\
& +L e_{n-1(j)}+L\left\|x_{n-1}-x_{n}\right\| .
\end{aligned}
$$

Using (2.3) in (2.4) for $j=1$ we have

$$
\begin{aligned}
\left\|x_{n}-T_{1} x_{n}\right\| \leq & e_{n(1)}+\left(L^{2}+L\right)\left\|x_{n}-x_{n-1}\right\|+L e_{n-1(1)} \\
\leq & e_{n(1)}+\left(L^{2}+L\right) e_{n-1(r)}+L e_{n-1(1)} \\
& +\left(L^{2}+L\right) c_{n-1(r)}\left\|U_{n-1(r)}-x_{n-1}\right\| .
\end{aligned}
$$


Using (2.2) and (2.3) in (2.4) for $j=2,3, \ldots, r$ we have

$$
\begin{aligned}
\left\|x_{n}-T_{j} x_{n}\right\| \leq & e_{n(j)}+\left(L^{2}+L\right)\left\|x_{n}-x_{n-1}\right\|+L^{2} e_{n(j-1)}+L^{2} e_{n-1(j-1)}+L e_{n-1(j)} \\
& +L^{2} c_{n(j-1)}\left\|u_{n(j-1)}-x_{n}\right\|+L^{2} c_{n-1(j-1)}\left\|x_{n-1}-u_{n-1(j-1)}\right\| \\
\leq & e_{n(j)}+\left(L^{2}+L\right) e_{n-1(r)}+L^{2} e_{n(j-1)}+L^{2} e_{n-1(j-1)}+L e_{n-1(j)} \\
& +\left(L^{2}+L\right) c_{n-1(r)}\left\|u_{n-1(r)}-x_{n-1}\right\|+L^{2} c_{n(j-1)}\left\|u_{n(j-1)}-x_{n}\right\| \\
& +L^{2} c_{n-1(j-1)}\left\|x_{n-1}-u_{n-1(j-1)}\right\| .
\end{aligned}
$$

This completes the proof of Lemma 2.2.

LEMmA 2.3. Let $C$ be a nonempty convex subset of a Banach space E. Let $\left\{T_{1}, T_{2}, \ldots\right.$, $\left.T_{r}\right\},\left\{u_{n(j)}\right\}$, and $\left\{x_{n}\right\}$ be as in Theorem 2.1 and let $\left\{a_{n(j)}\right\},\left\{b_{n(j)}\right\}$, and $\left\{c_{n(j)}\right\}$ satisfy conditions (i) and (ii) of Theorem 2.1. Then $\lim _{n \rightarrow \infty}\left\|x_{n}-x^{*}\right\|$ exists for all $x^{*} \in$ $\cap_{j=1}^{r} F\left(T_{j}\right)$.

Proof. Let $x^{*} \in \cap_{j=1}^{r} F\left(T_{j}\right)$. Since $\left\{u_{n(j)}\right\}_{n=1}^{\infty}$ and $\left\{k_{n}\right\}_{n=1}^{\infty}$ are bounded, there exists a constant $N>0$ such that $\sup _{n \in \mathbb{N}}\left\{\left\|u_{n(j)}-x^{*}\right\|: j=1,2, \ldots, r\right\} \leq N$ and $\sup _{n \in \mathbb{N}}\left\{1+k_{n}+\cdots+k_{n}^{r-1}\right\} \leq N$. Then, we have

$$
\begin{aligned}
\left\|x_{n+1}-x^{*}\right\|= & \left\|a_{n(r)} x_{n}+b_{n(r)} T_{r}^{n} U_{n(r-1)} x_{n}+c_{n(r)} u_{n(r)}-x^{*}\right\| \\
\leq & a_{n(r)}\left\|x_{n}-x^{*}\right\|+b_{n(r)}\left\|T_{r}^{n} U_{n(r-1)} x_{n}-x^{*}\right\|+c_{n(r)}\left\|u_{n(r)}-x^{*}\right\| \\
\leq & a_{n(r)}\left\|x_{n}-x^{*}\right\|+b_{n(r)} k_{n}\left\|U_{n(r-1)} x_{n}-x^{*}\right\|+N c_{n(r)} \\
= & a_{n(r)}\left\|x_{n}-x^{*}\right\|+b_{n(r)} k_{n} \\
& \times \| a_{n(r-1)}\left(x_{n}-x^{*}\right)+b_{n(r-1)}\left(T_{r-1}^{n} U_{n(r-2)} x_{n}-x^{*}\right) \\
& \quad+c_{n(r-1)}\left(u_{n(r-1)}-x^{*}\right) \|+N c_{n(r)} \\
\leq & {\left[1-b_{n(r)}+\left(1-b_{n(r-1)}\right) b_{n(r)} k_{n}\right]\left\|x_{n}-x^{*}\right\| } \\
& +b_{n(r)} b_{n(r-1)} k_{n}^{2}\left\|U_{n(r-2)} x_{n}-x^{*}\right\|+N c_{n(r)}+N^{2} c_{n(r-1)} \\
\vdots & \\
\leq & {\left[1-b_{n(r)}+\left(1-b_{n(r-1)}\right) b_{n(r)} k_{n}\right.} \\
& \left.\quad+\cdots+\left(1-b_{n(1)}\right) b_{n(r)} b_{n(r-1)} \cdots b_{n(2)} k_{n}^{r-1}+b_{n(r)} b_{n(r-1)} \cdots b_{n(1)} k_{n}^{r}\right] \\
& \cdot\left\|x_{n}-x^{*}\right\|+N\left(c_{n(r)}+N c_{n(r-1)}+\cdots+N c_{n(1)}\right) \\
= & {\left[1+b_{n(r)}\left(k_{n}-1\right)+b_{n(r)} b_{n(r-1)} k_{n}\left(k_{n}-1\right)\right.} \\
& \left.+\cdots+b_{n(r)} b_{n(r-1)} \cdots b_{n(1)}\left(k_{n}^{r-1}\right)\left(k_{n}-1\right)\right]\left\|x_{n}-x^{*}\right\|+\psi_{n} \\
\leq & {\left[1+\left(k_{n}-1\right)\left(1+k_{n}+\cdots+k_{n}^{r-1}\right)\right]\left\|x_{n}-x^{*}\right\|+\psi_{n} } \\
\leq & {\left[1+\left(k_{n}-1\right) N\right]\left\|x_{n}-x^{*}\right\|+\psi_{n} } \\
= & \left(1+\varphi_{n}\right)\left\|x_{n}-x^{*}\right\|+\psi_{n},
\end{aligned}
$$


for all $n \in \mathbb{N}$, where $\varphi_{n}:=\left(k_{n}-1\right) N$ and $\psi_{n}:=N\left(c_{n(r)}+N c_{n(r-1)}+\cdots+N c_{n(1)}\right)$. Since $\sum_{n=1}^{\infty}\left(k_{n}-1\right)<\infty$ and $\sum_{n=1}^{\infty} c_{n(j)}<\infty$ for each $j=1,2, \ldots, r$, we have $\sum_{n=1}^{\infty} \varphi_{n}<\infty$ and $\sum_{n=1}^{\infty} \psi_{n}<\infty$. Thus, $\lim _{n \rightarrow \infty}\left\|x_{n}-x^{*}\right\|$ exists by Lemma 1.1. This completes the proof of Lemma 2.3.

LEMMA 2.4. Under the hypotheses of Lemma 2.3, if $E$ is a uniformly convex Banach space, then there exists a continuous, strictly increasing and convex function $g: \mathbb{R}^{+} \rightarrow$ $\mathbb{R}^{+}$such that $g(0)=0$, and

$$
\sum_{n=1}^{\infty}\left[\sum_{j=1}^{r}\left(\prod_{l=j}^{r} \alpha_{n(l)}\right)\left(1-\alpha_{n(j)}\right) g\left(\left\|x_{n}-T_{j}^{n} U_{n(j-1)} x_{n}\right\|\right)\right]<\infty,
$$

where $\alpha_{n(j)}:=b_{n(j)}+c_{n(j)}$ for all $n \in \mathbb{N}$ and each $j=1,2, \ldots, r$.

Proof. Let $x^{*} \in \cap_{j=1}^{r} F\left(T_{j}\right)$. Lemma 2.3 and the hypotheses of Lemma 2.4 imply that $\left\{x_{n}-x^{*}\right\}_{n=1}^{\infty},\left\{u_{n(j)}\right\}_{n=1}^{\infty}$, and $\left\{k_{n}\right\}_{n=1}^{\infty}$ are bounded. Then, there exists a constant $d>0$ such that

$$
\cup_{j=1}^{r}\left\{T_{j}^{n} U_{n(j-1)} x_{n}-x^{*}\right\}_{n=1}^{\infty} \cup\left\{x_{n}-x^{*}\right\}_{n=1}^{\infty} \subseteq B_{d} .
$$

By Theorem 1.3, there exists a continuous, strictly increasing and convex function $g: \mathbb{R}^{+} \rightarrow \mathbb{R}^{+}$such that $g(0)=0$, and

$$
\|\lambda x+(1-\lambda) y\|^{2} \leq \lambda\|x\|^{2}+(1-\lambda)\|y\|^{2}-\lambda(1-\lambda) g(\|x-y\|)
$$

for all $x, y \in B_{d}$ and $\lambda \in[0,1]$. By inequality (2.10) we obtain the following estimate: for some constant $M$, we have

$$
\begin{aligned}
\left\|U_{n(j)} x_{n}-x^{*}\right\|^{2}= & \|\left(1-\alpha_{n(j)}\right)\left(x_{n}-x^{*}\right)+\alpha_{n(j)}\left(T_{j}^{n} U_{n(j-1)} x_{n}-x^{*}\right) \\
& -c_{n(j)}\left(T_{j}^{n} U_{n(j-1)} x_{n}-u_{n(j)}\right) \|^{2} \\
\leq & \left(\left\|\left(1-\alpha_{n(j)}\right)\left(x_{n}-x^{*}\right)+\alpha_{n(j)}\left(T_{j}^{n} U_{n(j-1)} x_{n}-x^{*}\right)\right\|\right. \\
& \left.\quad+c_{n(j)}\left\|\left(T_{j}^{n} U_{n(j-1)} x_{n}-u_{n(j)}\right)\right\|\right)^{2} \\
\leq & \left\|\left(1-\alpha_{n(j)}\right)\left(x_{n}-x^{*}\right)+\alpha_{n(j)}\left(T_{j}^{n} U_{n(j-1)} x_{n}-x^{*}\right)\right\|^{2}+c_{n(j)} M \\
\leq & \left(1-\alpha_{n(j)}\right)\left\|x_{n}-x^{*}\right\|^{2}+\alpha_{n(j)}\left\|T_{j}^{n} U_{n(j-1)} x_{n}-x^{*}\right\|^{2} \\
& -\alpha_{n(j)}\left(1-\alpha_{n(j)}\right) g\left(\left\|x_{n}-T_{j}^{n} U_{n(j-1)} x_{n}\right\|\right)+c_{n(j)} M \\
\leq & \left(1-\alpha_{n(j)}\right)\left\|x_{n}-x^{*}\right\|^{2}+\alpha_{n(j)} k_{n}^{2}\left\|U_{n(j-1)} x_{n}-x^{*}\right\|^{2} \\
& -\alpha_{n(j)}\left(1-\alpha_{n(j)}\right) g\left(\left\|x_{n}-T_{j}^{n} U_{n(j-1)} x_{n}\right\|\right)+c_{n(j)} M, \\
\left\|x_{n+1}-x^{*}\right\|^{2}= & \|\left(1-\alpha_{n(r)}\right)\left(x_{n}-x^{*}\right)+\alpha_{n(r)}\left(T_{r}^{n} U_{n(r-1)} x_{n}-x^{*}\right) \\
& -c_{n(r)}\left(T_{r}^{n} U_{n(r-1)} x_{n}-u_{n(r)}\right) \|^{2} \\
\leq & \left(1-\alpha_{n(r)}\right)\left\|x_{n}-x^{*}\right\|^{2}+\alpha_{n(r)} k_{n}^{2}\left\|U_{n(r-1)} x_{n}-x^{*}\right\|^{2} \\
& -\alpha_{n(r)}\left(1-\alpha_{n(r)}\right) g\left(\left\|x_{n}-T_{r}^{n} U_{n(r-1)} x_{n}\right\|\right)+c_{n(r)} M .
\end{aligned}
$$


By a repeated application of inequality (2.11) in (2.12), we obtain

$$
\begin{aligned}
\left\|x_{n+1}-x^{*}\right\|^{2} \leq & \left\|x_{n}-x^{*}\right\|^{2} \\
& +\alpha_{n(r)}\left(k_{n}^{2}-1\right)\left(1+\alpha_{n(r-1)} k_{n}^{2}+\cdots\right. \\
& \left.+\alpha_{n(r-1)} \alpha_{n(r-2)} \cdots \alpha_{n(1)} k_{n}^{2(r-1)}\right)\left\|x_{n}-x^{*}\right\|^{2} \\
& -\sum_{j=1}^{r}\left(\prod_{l=j}^{r} \alpha_{n(l)}\right)\left(1-\alpha_{n(j)}\right) g\left(\left\|x_{n}-T_{j}^{n} U_{n(j-1)} x_{n}\right\|\right) \\
& +\left(c_{n(r)}+k_{n}^{2} c_{n(r-1)}+\cdots+k_{n}^{2(r-1)} c_{n(1)}\right) M .
\end{aligned}
$$

Since $\sum_{n=1}^{\infty}\left(k_{n}-1\right)<\infty$, hence $\lim _{n \rightarrow \infty} k_{n}=1$, we may assume that $k_{n} \leq L$ for all $n \in \mathbb{N}$ and some constant $L$. Let $N=\max _{1 \leq j \leq r}\left\{L^{2 j}\right\} \geq 1$. Then

$$
\begin{aligned}
\left\|x_{n+1}-x^{*}\right\|^{2} \leq & \left\|x_{n}-x^{*}\right\|^{2}+\left(k_{n}-1\right)(N+1) r N d^{2}+M N \sum_{j=1}^{r} c_{n(j)} \\
& -\sum_{j=1}^{r}\left(\prod_{l=j}^{r} \alpha_{n(l)}\right)\left(1-\alpha_{n(j)}\right) g\left(\left\|x_{n}-T_{j}^{n} U_{n(j-1)} x_{n}\right\|\right)
\end{aligned}
$$

for all $n \in \mathbb{N}$. Transposing and summing from 1 to $m$ we have

$$
\begin{aligned}
\sum_{n=1}^{m}[ & \left.\sum_{j=1}^{r}\left(\prod_{l=j}^{r} \alpha_{n(l)}\right)\left(1-\alpha_{n(j)}\right) g\left(\left\|x_{n}-T_{j}^{n} U_{n(j-1)} x_{n}\right\|\right)\right] \\
& \leq\left\|x_{1}-x^{*}\right\|^{2}+(N+1) r N d^{2} \sum_{n=1}^{m}\left(k_{n}-1\right)+M N \sum_{n=1}^{m} \sum_{j=1}^{r} c_{n(j)} .
\end{aligned}
$$

Since $\sum_{n=1}^{\infty}\left(k_{n}-1\right)<\infty$ and $\sum_{n=1}^{\infty} c_{n(j)}<\infty$ for each $j=1,2, \ldots, r$, it follows that

$$
\sum_{n=1}^{\infty}\left[\sum_{j=1}^{r}\left(\prod_{l=j}^{r} \alpha_{n(l)}\right)\left(1-\alpha_{n(j)}\right) g\left(\left\|x_{n}-T_{j}^{n} U_{n(j-1)} x_{n}\right\|\right)\right]<\infty .
$$

This completes the proof of Lemma 2.4.

We now give the proof of Theorem 2.1.

Proof OF TheOrem 2.1. By Lemma 2.4 and condition (iii), we have

$$
(1-b) \sum_{n=1}^{\infty} \sum_{j=1}^{r} a^{r-j+1} g\left(\left\|x_{n}-T_{j}^{n} U_{n(j-1)} x_{n}\right\|\right)<\infty .
$$

Thus,

$$
\sum_{j=1}^{r} g\left(\left\|x_{n}-T_{j}^{n} U_{n(j-1)} x_{n}\right\|\right) \longrightarrow 0 \quad \text { as } n \rightarrow \infty .
$$

Since $g$ is a continuous and strictly increasing function with $g(0)=0$, we have $\lim _{n \rightarrow \infty}\left\|x_{n}-T_{j}^{n} U_{n(j-1)} x_{n}\right\|=0$ for each $j=1,2, \ldots, r$. Since $\left\{x_{n}-x^{*}\right\}$ and $\left\{u_{n(j)}\right\}$ are bounded. So we have

$$
\sup _{n \in \mathbb{N}}\left\{\left\|x_{n}-u_{n(j)}\right\|: j=1,2, \ldots, r\right\} \leq D
$$


for some constant $D>0$. Let $e_{n(j)}=\left\|x_{n}-T_{j}^{n} U_{n(j-1)} x_{n}\right\|$ and $L$ be as in the proof of Lemma 2.4. Then, by Lemma 2.2, we have

$$
\begin{aligned}
\left\|x_{n}-T_{1} x_{n}\right\| \leq & e_{n(1)}+\left(L^{2}+L\right) e_{n-1(r)}+L e_{n-1(1)}+\left(L^{2}+L\right) c_{n-1(r)} D \longrightarrow 0 \text { as } n \rightarrow \infty, \\
\left\|x_{n}-T_{j} x_{n}\right\| \leq & e_{n(j)}+\left(L^{2}+L\right) e_{n-1(r)}+L^{2} e_{n(j-1)}+L^{2} e_{n-1(j-1)}+L e_{n-1(j)} \\
& +\left(L^{2}+L\right) c_{n-1(r)} D+L^{2} c_{n(j-1)} D+L^{2} c_{n-1(j-1)} D \longrightarrow 0 \text { as } n \longrightarrow \infty
\end{aligned}
$$

for each $j=2,3, \ldots, r$. This completes the proof of Theorem 2.1.

THEOREM 2.5. Under the hypotheses of Theorem 2.1, if $E$ is a uniformly convex Banach space satisfying Opial's condition, then $\left\{x_{n}\right\}$ converges weakly to a common fixed point of $T_{1}, T_{2}, \ldots, T_{r}$.

Proof. Let $\omega_{w}\left(\left\{x_{n}\right\}\right)$ be the set of all weak subsequential limits of a bounded sequence $\left\{x_{n}\right\}$ in $C$. By Lemma 1.4 and Theorem 2.1, $\omega_{w}\left(\left\{x_{n}\right\}\right)$ is contained in $\cap_{j=1}^{r} F\left(T_{j}\right)$.

The remainder of the proof is similar to that of [9, Theorem 2.1], so the details are omitted.

REMARK 2.6. Theorem 2.5 generalizes [9, Theorem 2.1].

THEOREM 2.7. Under the hypotheses of Theorem 2.1. Suppose that $T_{1}^{m}$ is compact for some $m \in \mathbb{N}$. Then $\left\{x_{n}\right\}$ converges strongly to a common fixed point of $T_{1}, T_{2}, \ldots, T_{r}$.

Proof. As in the proof of [9, Theorem 2.2] by using Theorem 2.1 and Lemma 2.3, $\left\{x_{n}\right\}$ has a convergent subsequence $\left\{x_{n_{i}}\right\}$ such that $\lim _{i \rightarrow \infty} x_{n_{i}}=p$. Thus, by Theorem 2.1, we obtain that $T_{j} p=p$ for each $j=1,2, \ldots, r$. Hence, $p \in \cap_{j=1}^{r} F\left(T_{j}\right)$ and it follows from Lemma 2.3 that $\lim _{n \rightarrow \infty}\left\|x_{n}-p\right\|$ exists. Therefore, we conclude that $\lim _{n \rightarrow \infty}\left\|x_{n}-p\right\|=0$, completing the proof of Theorem 2.7.

REMARK 2.8. Theorem 2.7 generalizes [9, Theorem 2.2] and [6, Theorems 2 and 3].

LEMMA 2.9. Let $K$ be a compact convex subset of a normed space E. Suppose that $\alpha, \beta, \gamma \in[0,1]$ such that $\alpha+\beta+\gamma=1$. Then

$$
d(\alpha x+\beta y+\gamma z, K) \leq \alpha d(x, K)+\beta d(y, K)+\gamma d(z, K)
$$

for all $x, y, z \in E$ where $d(x, K):=\inf \{\|x-p\|: p \in K\}$.

Proof. Let $x, y, z \in E$. Since $K$ is compact, we have $d\left(x, p_{1}\right)=d(x, K), d\left(y, p_{2}\right)=$ $d(y, K)$, and $d\left(z, p_{3}\right)=d(z, K)$ for some $p_{1}, p_{2}, p_{3} \in K$. Since $K$ is convex so that $\alpha p_{1}+\beta p_{2}+\gamma p_{3} \in K$. Therefore,

$$
\begin{aligned}
d(\alpha x+\beta y+\gamma z, K) & \leq\left\|(\alpha x+\beta y+\gamma z)-\left(\alpha p_{1}+\beta p_{2}+\gamma p_{3}\right)\right\| \\
& \leq \alpha\left\|x-p_{1}\right\|+\beta\left\|y-p_{2}\right\|+\gamma\left\|z-p_{3}\right\| \\
& =\alpha d(x, K)+\beta d(y, K)+\gamma d(z, K)
\end{aligned}
$$

This completes the proof of Lemma 2.9. 
THEOREM 2.10. Under the hypotheses of Theorem 2.1. Suppose that there exists a nonempty compact convex subset $K$ of $E$ and some $\alpha \in(0,1)$ such that $d\left(T_{j} x, K\right) \leq$ $\alpha d(x, K)$ for all $x \in C$ and each $j=1,2, \ldots, r$. Then $\left\{x_{n}\right\}$ converges strongly to a common fixed point of $T_{1}, T_{2}, \ldots, T_{r}$.

Proof. For $n \in \mathbb{N}$ and $x \in C$ we have $d\left(T_{j}^{n} x, K\right) \leq \alpha^{n} d(x, K)$ for each $j=1,2, \ldots, r$. Since $\left\{u_{n(j)}\right\}_{n=1}^{\infty}$ is bounded for each $j=1,2, \ldots, r$ and $K$ is compact. Thus, there exists a constant $D>0$ such that

$$
\sup _{n \in \mathbb{N}}\left\{d\left(u_{n(j)}, K\right): j=1,2, \ldots, r\right\} \leq D
$$

Then, by Lemma 2.9, we have

$$
\begin{aligned}
d\left(x_{n+1}, K\right)= & d\left(a_{n(r)} x_{n}+b_{n(r)} T_{r}^{n} U_{n(r-1)} x_{n}+c_{n(r)} u_{n(r)}, K\right) \\
\leq & a_{n(r)} d\left(x_{n}, K\right)+b_{n(r)} d\left(T_{r}^{n} U_{n(r-1)} x_{n}, K\right)+c_{n(r)} d\left(u_{n(r)}, K\right) \\
\leq & a_{n(r)} d\left(x_{n}, K\right)+b_{n(r)} \alpha^{n} d\left(U_{n(r-1)} x_{n}, K\right)+c_{n(r)} D \\
\leq & \left(1-b_{n(r)}\right) d\left(x_{n}, K\right)+b_{n(r)} \alpha^{n} d\left(a_{n(r-1)} x_{n}+b_{n(r-1)} T_{r-1}^{n} U_{n(r-2)} x_{n}\right. \\
& \left.+c_{n(r-1)} u_{n(r-1)}, K\right)+c_{n(r)} D \\
\leq & {\left[1-b_{n(r)}+\left(1-b_{n(r-1)}\right) b_{n(r)} \alpha^{n}\right] d\left(x_{n}, K\right) } \\
& +b_{n(r)} b_{n(r-1)} \alpha^{2 n} d\left(U_{n(r-2)} x_{n}, K\right)+\left(c_{n(r-1)}+c_{n(r)}\right) D \\
\vdots & \\
\leq & {\left[1-b_{n(r)}\left(1-\alpha^{n}\right)\left(1+b_{n(r-1)} \alpha^{n}+\cdots+b_{n(r-1)} b_{n(r-2)} \cdots b_{n(1)} \alpha^{(r-1) n}\right)\right] } \\
d & \left(x_{n}, K\right)+\left(c_{n(1)}+c_{n(2)}+\cdots+c_{n(r)}\right) D \\
\leq & {\left[1-a\left(1-\alpha^{n}\right)\left(1+a \alpha^{n}+\cdots+a^{r-1} \alpha^{(r-1) n}\right)\right] d\left(x_{n}, K\right)+D \sum_{j=1}^{r} c_{n(j)} . }
\end{aligned}
$$

Let $\delta_{n}=a\left(1-\alpha^{n}\right)\left(1+a \alpha^{n}+\cdots+a^{r-1} \alpha^{(r-1) n}\right)$. Since $\lim _{n \rightarrow \infty} \delta_{n}=a$ and $0<a<1$, then there exists a real number $N_{0} \geq 1$ such that $\delta_{n}<1$ for all $n \geq N_{0}$. Since $\sum_{n=1}^{\infty} \delta_{n}=$ $\infty$ and $\sum_{n=1}^{\infty} \sum_{j=1}^{r} c_{n(j)}<\infty$, then by Lemma 1.2 , we have $\lim _{n \rightarrow \infty} d\left(x_{n}, K\right)=0$. Since $K$ is compact, this is easily seen to imply that $\left\{x_{n}\right\}$ has a convergent subsequence $\left\{x_{n_{i}}\right\}$ such that $\lim _{i \rightarrow \infty} x_{n_{i}}=p$. The rest of the proof is identical to the related part of the proof of Theorem 2.7.

REMARK 2.11. Theorem 2.10 generalizes [9, Theorem 2.4].

ACKNowledgement. This research was supported by the National Science Council of Republic of China, Project No. 90-2115-M-149-002.

\section{REFERENCES}

[1] K. Goebel and W. A. Kirk, A fixed point theorem for asymptotically nonexpansive mappings, Proc. Amer. Math. Soc. 35 (1972), 171-174. MR 45\#7552. Zbl 256.47045. 
[2] J. Górnicki, Weak convergence theorems for asymptotically nonexpansive mappings in uniformly convex Banach spaces, Comment. Math. Univ. Carolin. 30 (1989), no. 2, 249-252. MR 90g:47097. Zbl 686.47045.

[3] Z. Huang, Mann and Ishikawa iterations with errors for asymptotically nonexpansive mappings, Comput. Math. Appl. 37 (1999), no. 3, 1-7. MR 2000a:47118. Zbl 942.47046.

[4] Z. Opial, Weak convergence of the sequence of successive approximations for nonexpansive mappings, Bull. Amer. Math. Soc. 73 (1967), 591-597. MR 35\#2183. Zbl 179.19902.

[5] M. O. Osilike and S. C. Aniagbosor, Weak and strong convergence theorems for fixed points of asymptotically nonexpansive mappings, Math. Comput. Modelling 32 (2000), no. 10, 1181-1191. MR 2001h:47089. Zbl 01581388.

[6] B. E. Rhoades, Fixed point iterations for certain nonlinear mappings, J. Math. Anal. Appl. 183 (1994), no. 1, 118-120. MR 95e:47082. Zbl 807.47045.

[7] J. Schu, Iterative construction of fixed points of asymptotically nonexpansive mappings, J. Math. Anal. Appl. 158 (1991), no. 2, 407-413. MR 92d:47072. Zbl 734.47036.

[8] On a theorem of C. E. Chidume concerning the iterative approximation of fixed points, Math. Nachr. 153 (1991), 313-319. MR 93b:47123. Zbl 796.47047.

[9] _ Weak and strong convergence to fixed points of asymptotically nonexpansive mappings, Bull. Austral. Math. Soc. 43 (1991), no. 1, 153-159. MR 91k:47136. Zbl 709.47051.

[10] H. K. Xu, Inequalities in Banach spaces with applications, Nonlinear Anal. 16 (1991), no. 12, 1127-1138. MR 92e:47126. Zbl 757.46033.

[11] Y. Xu, Ishikawa and Mann iterative processes with errors for nonlinear strongly accretive operator equations, J. Math. Anal. Appl. 224 (1998), no. 1, 91-101. MR 99g:47144. Zbl 936.47041.

Jui-Chi Huang: Department of General Education, Kuang Wu Institute of TeChNOLOGY, Peito, TAIPEI, TAIWAN 11271, R.O.C.

E-mail address: juichi@kwi t. edu. tw 


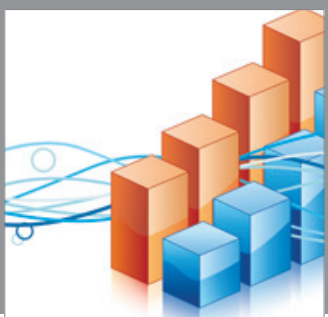

Advances in

Operations Research

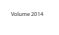

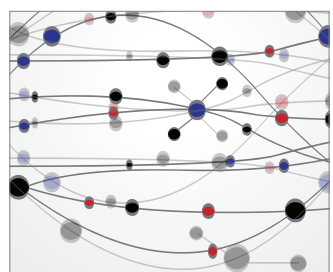

\section{The Scientific} World Journal
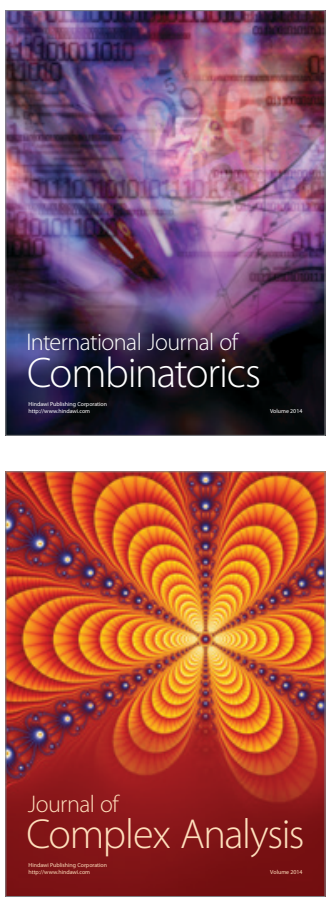

International Journal of

Mathematics and

Mathematical

Sciences
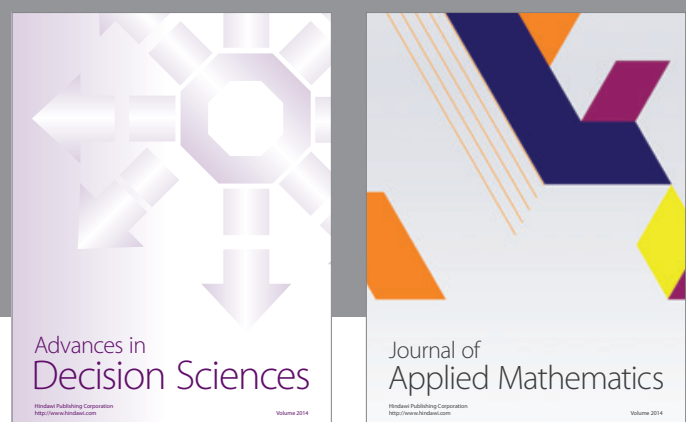

Journal of

Applied Mathematics
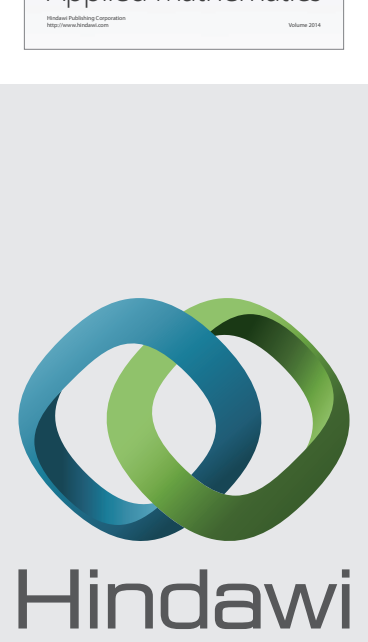

Submit your manuscripts at http://www.hindawi.com
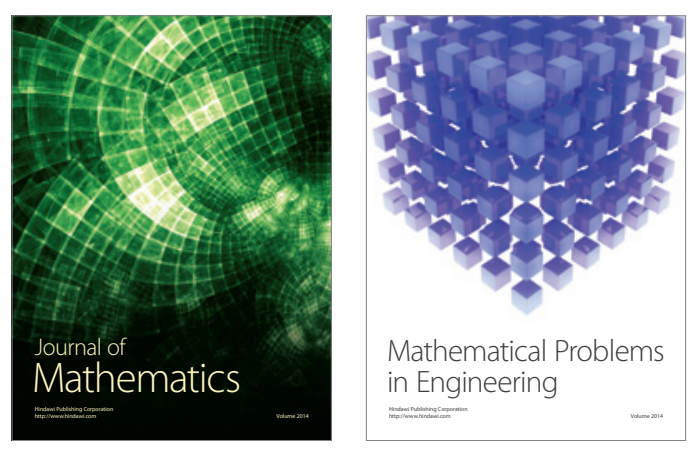

Mathematical Problems in Engineering
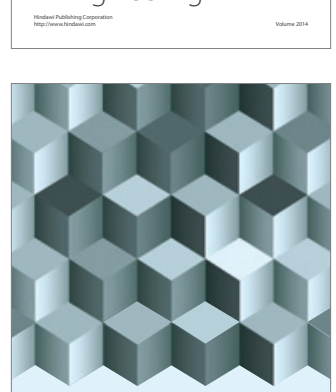

Journal of

Function Spaces
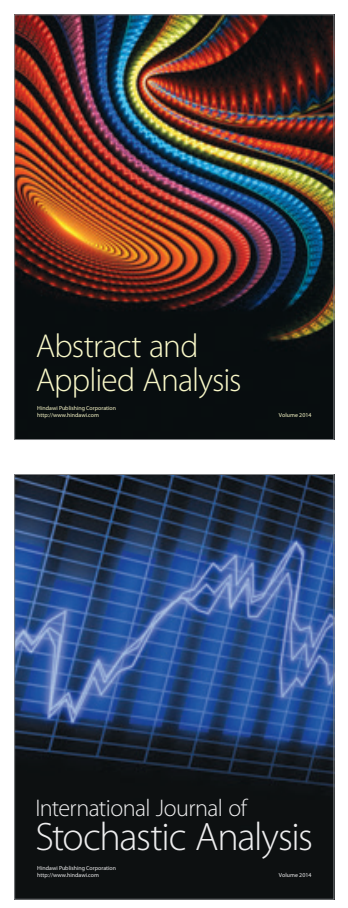

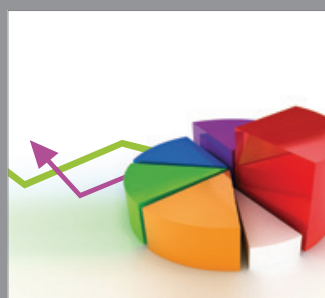

ournal of

Probability and Statistics

Promensencen
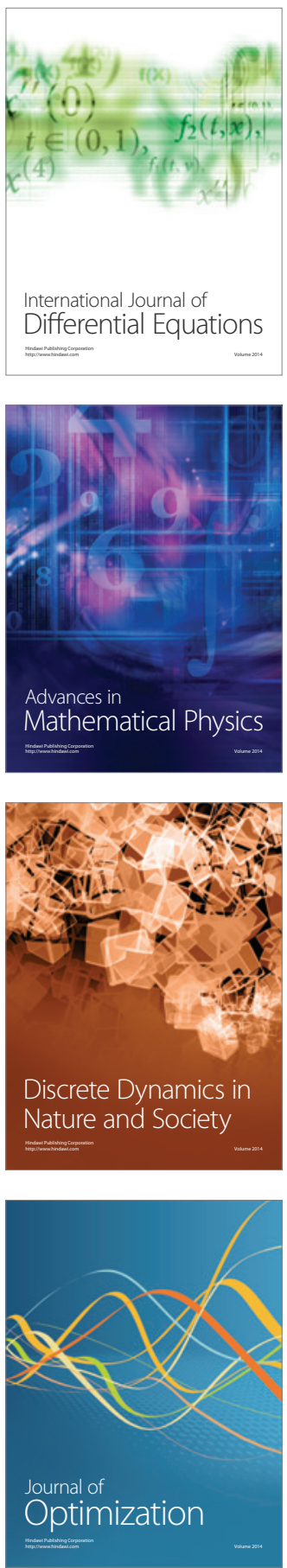